\title{
DISERTACIONES
}

ESTUDIOS

Para citar este artículo: Fonseca Matera, E. N., \& Pellegrini, P. A. (2021). El rol de los medios de comunicación como iniciadores de una controversia sociocientífica: el caso de la crotoxina en Argentina. Anuario Electrónico de Estudios en Comunicación Social "Disertaciones", 14(2), 1-19.

\section{EL ROL DE LOS MEDIOS DE COMUNICACIÓN COMO INICIADORES DE UNA CONTROVERSIA SOCIOCIENTÍFICA: EL CASO DE LA CROTOXINA EN ARGENTINA}

The Role of the Media as Starters of a Socio-Scientific Controversy: The Crotoxin Case in Argentina

O papel da mídia como iniciadora de uma controvérsia sociocientífica:

o caso da crotoxina na Argentina

Emilio Nahuel Fonseca Matera, Instituto de Estudios sobre la Ciencia y la Tecnología, Universidad Nacional de Quilmes-CIC-BA (Argentina) efonsecamatera@uvq.edu.ar

Pablo Ariel Pellegrini, CONICET e Instituto de Estudios sobre la Ciencia y la Tecnología, Universidad Nacional de Quilmes-CIC-BA (Argentina) ppellegrini@unq.edu.ar

Recibido: 15 de octubre de 2020

Aprobado: 2 de marzo de 2021

Fecha de prepublicación: 8 de junio de 2021 


\section{RESUMEN}

Los estudios sociales de la ciencia han prestado particular atención a las controversias científicas; pero no necesariamente en relación con el rol de los medios de comunicación, el cual, con frecuencia, es analizado como una cuestión de comunicación pública de la ciencia. En este trabajo, sin embargo, se vinculan ambas dimensiones, analizando el rol de los medios masivos de comunicación en el debate que giró en torno a la crotoxina como posible cura contra el cáncer, debate que ocupó el centro de la arena pública a mediados de 1986, en Argentina. El caso se reconstruyó a partir del análisis de los periódicos y programas televisivos de la época, a través de entrevistas a los actores centrales involucrados en el conflicto y mediante fuentes secundarias. Al analizar cómo llega una determinada situación del ámbito científico a dominar la escena pública, este trabajo busca contribuir a la comprensión de un fenómeno de creciente relevancia en las sociedades actuales: de qué manera los medios masivos de comunicación inciden en las controversias vinculadas a la ciencia, al instalar en la agenda pública una determinada forma de entender un tema científico.

Palabras clave: controversias sociocientíficas; crotoxina; medios de comunicación.

\section{ABSTRACT}

Scientific controversies have received special attention from the social studies of science, although not necessarily associating them with the role played by the media, frequently assessed as a matter of public communication of science. In this work, however, we will link both dimensions through the analysis of the role of the mass media concerning the discussion revolving around crotoxin as a potential cure for cancer, a debate that held the public spotlight by mid-1986 in Argentina. The case has been reconstructed from the analyses of newspapers and television programs of that time, through interviews with the central actors involved in the conflict, and based on secondary sources. By assessing how the scientific field came to dominate the public arena, this work aims at contributing to the understanding of rapidly growing phenomena in present-day societies: how the mass media influences science-related controversies by setting up a scientific issue on the public agenda.

Keywords: Socio-scientific controversies; crotoxin; media.

\section{RESUMO}

Os estudos sociais da ciência têm dado atenção especial às controvérsias científicas, mas não necessariamente vinculando-as ao papel da mídia, que muitas vezes é vista como uma questão de comunicação pública da ciência. Neste trabalho, entretanto, vincularemos as duas dimensões, analisando o papel da mídia de massa no debate que girava em torno da crotoxina como possível cura para o câncer, debate que ocupou o centro da arena pública em meados de 1986 na Argentina. O caso é reconstruído a partir da análise dos jornais e programas de televisão da época, por meio de entrevistas com os atores centrais envolvidos no conflito e por meio de fontes secundárias. Ao analisar como determinada situação no campo científico passa a dominar a cena pública, este trabalho busca 


\section{DISERTACIONES}

ESTUDIOS

Comunicación y controversias sociocientificas en salud y medio ambiente

ISSN: $1856-9536$

Doi: https://doi.org/10.12804/revistas.urosario.edu.co/disertaciones/a.9774

Volumen 14, Número 2 / Julio-diciembre 2021

Versión PDF para imprimir desde

http://revistas.urosario.edu.co/index.php/disertaciones

contribuir para a compreensão de um fenômeno cada vez mais relevante nas sociedades atuais: como os meios de comunicação influenciam as controvérsias relacionadas à ciência ao instalar uma determinada forma de compreensão de um tema científico na agenda pública.

Palavras-chave: controvérsias sociocientíficas; crotoxina; mídia.

\section{Introducción}

Las relaciones entre los medios masivos de comunicación y la ciencia han sido objeto de numerosos estudios que han analizado los procesos de comunicación de la ciencia, los modos de conceptualizar el vínculo entre el público y los expertos, la imagen de la ciencia que se transmite, entre otras cuestiones (Lewenstein, 1995; Schäfer, 2011; Rödder et al., 2012). Desde los estudios sociales de la ciencia, también ha despertado interés analizar el rol de los medios de comunicación en la generación y configuración de debates públicos con componentes científicos, esto es, la influencia de los medios de comunicación en las controversias sociocientíficas. En esa línea de análisis se inscribe nuestro trabajo. Siguiendo a Goodell (1987), se pueden analizar distintas formas de influencia de los medios en una controversia científica: la imagen cultural de la ciencia y de los científicos que transmiten los medios, el interés de los medios por mostrar noticias conflictivas acerca de la ciencia, el modo en que los medios recurren a cierto tipo de información sobre un tema y, finalmente -aunque vinculado con lo anterior-, la influencia de los medios en trasladar una discusión científica a la arena pública. Esta última cuestión constituye el centro de análisis de nuestro trabajo, en particular, el interés por comprender el rol de los medios masivos de comunicación en instalar como un tema controversial algo que dentro de la comunidad científica no se consideraba mayoritariamente como tal.

Nuestro trabajo se despliega en torno a una controversia sociocientífica sobre un tema de salud que generó un gran debate público: el caso de la crotoxina como posible cura contra el cáncer, debate que ocupó el centro de la arena pública a mediados de 1986, en Argentina. La crotoxina es un complejo proteico presente en el veneno de la serpiente de cascabel sudamericana. Durante algún tiempo, el mecanismo de acción y la citotoxicidad (destrucción de células) de este tipo de moléculas los estudiaron diversos investigadores en el campo de la bioquímica, aunque sin resultados prometedores en cuanto a su uso terapéutico. Su utilización en personas no se encontraba autorizado; sin embargo, durante la década de 1980 unos médicos, en colaboración con un investigador del Consejo Nacional de Investigaciones Científicas y Técnicas (Conicet), suministraron crotoxina a pacientes con diversas patologías, mayormente relacionadas con diferentes tipos de cáncer. Esa situación cobró estado público cuando los médicos acudieron a un programa de televisión para denunciar que no conseguían más crotoxina para 


\section{DISERTACIONES}

ESTUDIOS

Comunicación y controversias sociocientificas en salud y medio ambiente

ISSN: $1856-9536$

Doi: https://doi.org/10.12804/revistas.urosario.edu.co/disertaciones/a.9774

Volumen 14, Número 2 / Julio-diciembre 2021

Versión PDF para imprimir desde

http://revistas.urosario.edu.co/index.php/disertaciones

suministrar a sus pacientes. A partir de allí se desplegó una controversia pública con los partícipes de esa investigación como defensores del uso terapéutico de la crotoxina, buena parte de la sociedad expectante de encontrar allí una cura contra el cáncer y organismos científicos que negaban la evidencia en favor de la crotoxina.

La metodología empleada en este trabajo consiste en un estudio de caso en torno a dicha controversia. Se reconstruyó a partir del análisis de los periódicos y programas televisivos de la época, a través de entrevistas a los actores centrales involucrados en el conflicto, y mediante fuentes secundarias. Las notas periodísticas analizadas provienen de los diarios La Nación, La Prensa, Clarín y Tiempo Argentino. Con excepción del caso del periódico Tiempo Argentino, el resto de los medios de prensa escrita se eligieron según la magnitud de alcance nacional. La Nación, Clarín y La Prensa eran parte de los periódicos de alcance nacional de mayor tirada a comienzos de la década de 1980 (Borelli, 2011, p. 29). En cuanto a Tiempo Argentino, este periódico fue el único en cubrir la primera aparición pública de los protagonistas del caso y, por ello, justificamos su elección. Respecto de las entrevistas a los actores centrales involucrados en el conflicto, se le realizó una en profundidad a un actor clave de la problemática analizada: se trata de la productora del programa La Noticia Rebelde, que estuvo a cargo de la entrevista que allí se realizó por primera vez a los médicos que defendían el uso de la crotoxina (Igal, 2019).

De este modo, el presente trabajo pretende contribuir a la comprensión de un fenómeno de creciente relevancia en las sociedades actuales: de qué manera los medios masivos de comunicación inciden en las controversias vinculadas con la ciencia, al instalar en la agenda pública una determinada forma de entender un tema científico. Para ello, comenzaremos por presentar el problema general de los medios masivos de comunicación como iniciadores de controversias sociocientíficas. Luego abordaremos el análisis del caso particular de la crotoxina, lo que nos llevará a reconstruir la controversia que tuvo lugar en Argentina en 1986. En una tercera sección, analizaremos específicamente el rol de los medios de comunicación en el caso de la controversia sobre la crotoxina. Finalmente, brindaremos unas conclusiones de nuestro análisis en el marco del interés por comprender el papel de los medios masivos de comunicación en las controversias sociocientíficas.

\section{Los medios de comunicación en la construcción de un problema público sobre temas científicos}

Herbert Blumer (1971), hace ya medio siglo, señaló que los problemas sociales no pueden ser considerados meros reflejos de un problema objetivo que aqueja a la sociedad, sino que forman parte de un proceso de definición colectivo. Blumer cuestionaba así los abordajes que, frente a un debate público sobre un problema que afecta a una parte de la sociedad, se limitaban a presentar lo que serían las condiciones objetivas de ese problema. Pues hay infinidad de situaciones objetivas que pueden resultar dañinas para la sociedad y, sin embargo, en la arena pública solo se debaten algunos temas puntuales. De modo que los debates sobre problemas sociales no deberían naturalizarse, y la indagación sociológica debería problematizar cómo es que un tema llega a ser seleccionado, construido o presentado en la arena pública como un problema.

En ese sentido, en un trabajo ya clásico, Joseph Gusfield (2014) abordó un problema cuya naturaleza objetiva parecía indiscutible -el problema de los conductores alcoholizados-, para demostrar que incluso allí también operó un proceso social que lo llevó a convertirse en un problema público particular. Gusfield demuestra que las 


\section{DISERTACIONES}

ESTUDIOS

Comunicación y controversias sociocientíficas en salud y medio ambiente

ISSN: 1856-9536

Doi: https://doi.org/10.12804/revistas.urosario.edu.co/disertaciones/a.9774

Volumen 14, Número 2 / Julio-diciembre 2021

Versión PDF para imprimir desde

http://revistas.urosario.edu.co/index.php/disertaciones

muertes en accidentes de tránsito cuando hay consumo de alcohol pueden verse como un problema que se origina en el hecho de que los conductores están ebrios, pero que también podría ser pensado como un problema cuya causa radica en los insuficientes dispositivos de seguridad en el diseño de los automóviles, o en el diseño de las autopistas, o debido a una cultura que estimula el consumo de alcohol, o incluso podría no haber sido considerado un problema público. Es decir, no hay una forma natural, objetiva, de que un determinado tema sea asumido como problema público. El punto es que, ante una multiplicidad de problemas sociales complejos, solo algunos llegan a la arena pública y lo hacen de un modo muy acotado.

Continuando con esta línea de análisis, Hilgartner y Bosk (1988) llegaron a la conclusión de que la definición colectiva de los problemas sociales no ocurre en un espacio indefinido como sería la "opinión pública", sino que hay arenas públicas específicas donde a los problemas sociales se les da forma y desarrollo. Uno de estos ámbitos específicos que tiene la capacidad de instalar un tema en la agenda pública es el de los medios de comunicación. A partir de allí, el análisis del rol de los medios de comunicación en la construcción de los problemas sociales se transforma en un tema relevante de estudio. En particular, a nosotros nos interesa indagar por el rol de los medios masivos de comunicación en la construcción de controversias sociocientíficas.

Brian Martin (2014) observa que los medios pueden tener tres roles distintos en las controversias: 1) pueden oficiar como un transmisor de información científica hacia un público más amplio (aunque, por supuesto, como un transmisor no neutral, susceptible a sesgos y presiones); 2) los medios pueden desplegar un rol activo, mostrando afinidad hacia una de las posiciones en conflicto, ya sea por compromisos ideológicos o intereses empresariales, y 3) el que más nos interesa destacar, pues se encadena con la perspectiva teórica que venimos desarrollando: los medios de comunicación, dice Martin (2014, p. 129), pueden afectar las controversias científicas a través de la instalación de una agenda (agenda-setting). Esto es, al mostrar historias y noticias sobre un tema, los medios instalan la idea de que ese tema merece la atención, de que es más importante que otros.

Es relevante entonces entender cómo llega una determinada situación del ámbito científico a dominar la escena pública. Pellegrini (2019, pp. 177-181) muestra dos casos de controversias sociocientíficas que se disparan en la arena pública cuando un científico acude a un medio de comunicación masivo para informar a la población -antes que a sus colegas de la comunidad científica - sobre lo que considera un problema. En 1998, el Dr. Pusztai formaba parte de un proyecto que involucraba a un conglomerado de instituciones británicas y cuyo objetivo era generar plantas genéticamente modificadas y medir su impacto sobre el medio ambiente. Como especialista en nutrición, Pusztai tenía una función acotada y específica en el proyecto, vinculada a realizar estudios nutricionales en ratas. En agosto de ese año, cuando el proyecto aún estaba lejos de concluir, Pusztai acudió a uno de los programas televisivos más vistos en Gran Bretaña en la época, Word in Action, el cual se caracterizaba por hacer periodismo de investigación. Allí, frente a las cámaras de televisión, Pusztai afirmó que él jamás comería un alimento derivado de cultivos transgénicos y que, de hecho, consideraba "muy injusto usar a nuestros ciudadanos como conejillos de indias". A partir de ese momento, la controversia en torno a alimentos derivados de cultivos transgénicos tomó el centro de la escena pública, desde periódicos que mencionaban el tema cotidianamente hasta políticos que pedían moratorias para la comercialización de transgénicos. Pero la comunidad científica no fue tan entusiasta en respaldar las palabras de Pusztai. Si bien algunos investigadores - de temas muy diversos, la mayoría no vinculados al problema en cuestión- salieron a respaldarlo públicamente, muchos le recriminaron no haber esperado a concluir su investigación y no haberla puesto a consideración de la comunidad científica. 


\section{DISERTACIONES}

ESTUDIOS

Comunicación y controversias sociocientificas en salud y medio ambiente

ISSN: $1856-9536$

Doi: https://doi.org/10.12804/revistas.urosario.edu.co/disertaciones/a.9774

Volumen 14, Número 2 / Julio-diciembre 2021

Versión PDF para imprimir desde

http://revistas.urosario.edu.co/index.php/disertaciones

Se realizó una auditoría sobre el trabajo de Pusztai y las conclusiones fueron que sus resultados eran muy inconsistentes. Finalmente, en junio de 1999, la Royal Society de Londres concluyó que el trabajo de Pusztai era deficiente en cuanto a su diseño, ejecución y análisis, y que no debían sacarse conclusiones de este. Pero a pesar de que la comunidad científica mostraba así un contundente rechazo a las conclusiones que Pusztai había vertido en la televisión, eso no clausuró una controversia que, por cierto, trascendía el ámbito de la comunidad científica.

Otro caso con características similares tuvo lugar en Argentina en 2009, cuando el Dr. Carrasco -médico especializado en temas de biología del desarrollo y que se encontraba realizando una investigación sobre tejidos de anfibios a los que se les suministraba glifosato- concedió una entrevista a un periódico de alcance nacional en la que sostuvo que no debería usarse el glifosato como herbicida en la agricultura, porque tenía efectos negativos en la morfología de los embriones. También en este caso Carrasco fue cuestionado por haber acudido a un medio de comunicación masivo en lugar de haber discutido sus resultados con la comunidad científica (de hecho, su investigación salió publicada en un artículo académico recién un año y medio después de haber brindado la entrevista) y por haber extrapolado un ensayo específico a una situación muy distinta. Por su parte, un consejo científico interdisciplinario fue convocado para analizar la peligrosidad del glifosato a partir del escándalo que se había generado y su conclusión fue que bajo condiciones de uso responsable implicaba un bajo riesgo para la salud o el ambiente. Sin embargo, tampoco ese consejo científico clausuró un debate que a partir de ese momento había tomado el centro de la arena pública.

Un rasgo común en estos casos es que el hecho en debate se transforma en un problema público aun antes de ser una discusión científica:

Cuando un científico acude a un medio masivo de comunicación, instala un discurso de gran autoridad (dado el reconocimiento social que suele recibir el ámbito científico) en un espacio de mucha incidencia en la formulación de problemas públicos. Por otro lado, la comunidad científica reacciona a esa aparición mediática con un interrogante muy específico: se pregunta si ese tema ya había sido demostrado previamente en los canales propios de la comunidad científica (las revistas especializadas). Si el investigador está comunicando en un medio masivo algo que aún no había expuesto ante la comunidad científica, eso es asumido como una falta, pues sus colegas no tuvieron la oportunidad de validar ese conocimiento. Por su parte, el argumento del científico que actuó de esa manera suele consistir en decir que estaba ante una urgencia, que debía alertar sobre un problema a la población general en base a los resultados observados. (Pellegrini, 2019, p. 178)

David Harker (2015) considera que una controversia científica es una "controversia creada" cuando lo que se presenta en la arena pública no coincide con la evaluación que hacen los expertos. Por su parte, Goodell (1987, p. 590) observa que en el tratamiento de las controversias los medios suelen generar un patrón de dependencia en relativamente pocas fuentes científicas, lo cual lleva a los medios a magnificar la perspectiva de algunos científicos, en lugar de ofrecer un panorama más completo de los expertos en el tema.

En función de lo expuesto, consideramos que la caracterización del rol de los medios de comunicación como iniciadores de una controversia sociocientífica puede realizarse teniendo en cuenta: 1) cómo llega el tema a instalarse en la agenda pública y bajo qué perspectiva se presenta; 2) cuál es el grado de desarrollo y discusión que tenía ese tema en la comunidad científica antes de llegar a la arena pública (si se trata de una investigación incipiente o si sus resultados ya han atravesado algún tipo de validación colectiva en la comunidad científica);3) cuán representativas 


\section{DISERTACIONES}

ESTUDIOS

Comunicación y controversias sociocientificas en salud y medio ambiente

ISSN: $1856-9536$

Doi: https://doi.org/10.12804/revistas.urosario.edu.co/disertaciones/a.9774

Volumen 14, Número 2 / Julio-diciembre 2021

Versión PDF para imprimir desde

http://revistas.urosario.edu.co/index.php/disertaciones

son las voces científicas que aparecen en los medios de comunicación en relación con el tema controversial (en qué fuentes de información se basan los medios para presentar el tema, cuán variadas, representativas o balanceadas son), y 4) cuál es la evaluación que hace la mayoría de la comunidad científica sobre el tema y si difiere con el modo en que se presenta la evaluación del tema en la arena pública (lo que permitiría distinguir entre una controversia creada o no).

\section{La controversia sobre la crotoxina}

Analizamos a continuación el rol de los medios de comunicación en la controversia sobre la crotoxina, para lo cual reconstruiremos primero el desarrollo del conflicto.

La crotoxina es el componente neurotóxico del veneno de la serpiente de cascabel sudamericana (Crotalus durissus terrificus). Aislada y cristalizada por primera vez en 1938 por los bioquímicos Karl Heinrich Slotta y Heinz Fraenkel-Conrat, la crotoxina posee actividad enzimática y es reconocida su citotoxicidad (daña células del organismo). Si bien desde la década de 1930 el veneno de diferentes crotálidos, junto al de otras familias y subfamilias de serpientes, había sido estudiado por sus posibles efectos antitumorales (Essex \& Priestley, 1931; Calmette et al., 1933; Monaelesser \& Taguet, 1933; Braganca et al., 1965; Cotte et al., 1972; entre otros), lo cierto es que las investigaciones sobre posibles nuevos tratamientos en la terapéutica del cáncer no proliferaron mucho más allá por el camino del estudio de los venenos de serpiente de cascabel, o de la crotoxina en particular. Por lo menos, no hasta la década de 1980. En 1979, el doctor en bioquímica Juan Carlos Vidal, un investigador argentino perteneciente al Conicet, halló de forma casual -según su testimonio- que la crotoxina "causaba lisis celular en las células tumorales y respetaba a las células normales" (Barrios Medina \& Dellacha, 2007, p. 68; "Un recurso de amparo para que se autorice la aplicación de crotoxina", 1986). Vidal trabajaba en el Instituto de Neurobiología (IDNEU), dirigido por el Dr. Juan H. Tramezzani, y se encontraba a cargo del serpentario perteneciente a uno de los centros de investigación que se ubicaba en el mismo edificio.

A principios de 1980, el Dr. Vidal comenzó a elaborar y suministrar una sustancia denominada complejo crotoxina $A$ y $B$ a seres humanos como tratamiento para diversas patologías, mayormente relacionadas con diferentes tipos de cáncer (Schulz \& Katime, 2003, p. 39). En 1983, Vidal proporcionó fracciones de crotoxina a un médico "que lo había solicitado para una paciente de la Clínica del Sol que padecía un melanoma con metástasis cerebrales [sic]" (Barrios Medina \& Dellacha, p. 21). En 1985, conoció al médico tratante de la paciente de la Clínica del Sol, Guillermo Hernández Plata, quien junto con los médicos Luis Costa y Carlos Coni Molina (este último era médico con especialización en oncología), comenzó a vincularse con Vidal y a trabajar con el complejo a base de crotoxina para el tratamiento de pacientes con distintos tipos de cáncer de forma experimental en un consultorio privado. Los tres médicos se encargaban del diagnóstico y el seguimiento de pacientes a quienes se les administraba el complejo crotoxina; mientras que Vidal se ocupaba de la producción y el suministro del complejo (Barrios Medina \& Dellacha, 2007, p. 22).

Dicha dinámica se mantuvo sin mayores percances hasta 1986. A principios de ese año, Juan H. Tramezzani, director del IDNEU, solicitó a Vidal "explicaciones sobre movimientos extraños observados en relación con el laboratorio de venenos" (Braun, 1989, p. 71). Vidal informó que se estudiaba el efecto de la crotoxina en pacientes oncológicos terminales y que se contaba con el aval de las autoridades correspondientes, aunque no presentó el 


\section{DISERTACIONES}

ESTUDIOS

Comunicación y controversias sociocientíficas en salud y medio ambiente

ISSN: 1856-9536

Doi: https://doi.org/10.12804/revistas.urosario.edu.co/disertaciones/a.9774

Volumen 14, Número 2 / Julio-diciembre 2021

Versión PDF para imprimir desde

http://revistas.urosario.edu.co/index.php/disertaciones

aval en concreto. Tramezzani concertó con Vidal una reunión con la presencia de los tres médicos en la que estos expusieron resultados prometedores de las investigaciones desarrolladas hasta ese momento (Barrios Medina \& Dellacha, 1986, p. 23). En abril de 1986, Vidal entregó a Tramezzani una monografía titulada Complejo crotoxina A y $B$ en el tratamiento del cáncer, que contenía la descripción de la investigación básica sobre los efectos antitumorales del complejo crotoxina y en la cual se incluía información sobre la realización de estudios in vitro sobre tejidos tumorales, procedimientos experimentales sobre roedores y ensayos clínicos en pacientes afectados por distintos tipos de cáncer (Braun, 1989, p. 71). En mayo de 1986, Vidal y los médicos Hernández Plata, Costa y Coni Molina inscribieron la monografía en el Registro de la Propiedad Intelectual y solicitaron "la patente de obtención y aplicación del Complejo Crotoxina A y B en la terapéutica del cáncer”, lo que implicó un conflicto con el marco regulatorio de derechos sobre patentes del Conicet (Barrios Medina \& Dellacha, 2007, p. 24). Al haber sido este un desarrollo llevado a cabo en un laboratorio que mantenía un convenio con el Conicet (con el correspondiente uso de sus instalaciones y materiales) y por un investigador del propio Consejo, debía quedar bajo la normativa de vinculación dictaminada por la misma institución. Esta normativa implicaba la cesión de derechos por parte del investigador (Ley 20464 de 1973).

El 15 de junio de 1986, Juan Carlos Vidal partió hacia Estados Unidos para radicarse allí durante una estadía de investigación que había sido pactada con el Dr. Paul B. Sigler, de la Universidad de Chicago. El trabajo experimental que había sido encausado por Vidal junto a los médicos Hernández Plata, Costa y Coni Molina quedaba acéfalo o, por lo menos, sin su nexo particular con las autoridades y el personal del laboratorio donde era producida la crotoxina.

Desde 1985, los pacientes a quienes les era suministrada la droga fueron incrementándose con el correr del tiempo y, al parecer, debido a la divulgación realizada por pacientes anteriores y por los médicos tratantes. En su ausencia, Vidal había dejado a cargo de la producción y entrega del complejo a sus colaboradoras en el IDNEU. La situación comenzaba a agudizarse, en parte debido al incremento de pacientes en las puertas del instituto y debido a que el asunto comenzó a cobrar notoriedad entre los diversos investigadores de los centros que se ubicaban en el edificio (Barrios Medina \& Dellacha, 2007, pp. 27 y 28). Además, Tramezzani había tomado cuenta de que los estudios se realizaban en un consultorio médico privado y sin autorización alguna de Salud Pública ("Hubo fraude y ocultamiento en la preparación de la droga", 1986). Ante esta situación y tras no lograr acordar nuevas reuniones con los tres médicos, Tramezzani decidió suspender la entrega de crotoxina a los pacientes (De Ípola, 2002, p. 374).

En aquel contexto de tensión, el 7 de julio de 1986, los tres médicos se presentaron en el Conicet para denunciar la decisión de Tramezzani, convencidos de que la investigación contaba con el auspicio del consejo (Garrahan, 1995, pp. 14-17). Mantuvieron una reunión con Héctor Ciapuscio, secretario ejecutivo del Conicet, y le entregaron la monografía Complejo crotoxina $A$ y $B$ en el tratamiento del cáncer. La entrega de la monografía tenía por objeto mostrar la prueba del éxito experimental y clínico del tratamiento que estaban desarrollando los médicos, quienes, además, expresaron a Ciapuscio que "la presión de los pacientes por el reclamo era incontenible" (Barrios Medina \& Dellacha, 2007, p. 31). El martes 8 de julio, los médicos decidieron realizar una conferencia de prensa y anunciaron el descubrimiento de un tratamiento antineoplásico exitoso y explicaron las motivaciones que los llevaron a realizar esta presentación fuera del ámbito de la comunidad científica. Al día siguiente, una nueva presentación de los tres médicos, esta vez en el programa televisivo humorístico La Noticia Rebelde, culminó con el alcance masivo de la noticia a miles de hogares del país. 


\section{DISERTACIONES}

ESTUDIOS

Comunicación y controversias sociocientíficas en salud y medio ambiente

ISSN: 1856-9536

Doi: https://doi.org/10.12804/revistas.urosario.edu.co/disertaciones/a.9774

Volumen 14, Número 2 / Julio-diciembre 2021

Versión PDF para imprimir desde

http://revistas.urosario.edu.co/index.php/disertaciones

La novedad expuesta por los tres médicos causó un gran estupor y múltiples repercusiones en la esfera pública. Al día siguiente del anuncio, el Ministerio de Salud y Acción Social (msyAs) emitió un comunicado en el que expresaba su desconocimiento sobre el tratamiento divulgado por los médicos. En el comunicado, a su vez, el ministerio afirmaba la obligatoriedad de comunicar y solicitar una autorización al área de la Secretaría de Salud para la realización de investigaciones con fármacos y, por último, anunciaba la citación a los médicos Coni Molina, Costa y Hernández Plata ("Citarán a médicos por el supuesto hallazgo de una droga contra el cáncer", 1986). Por su parte, el Conicet decidió emitir un comunicado en la misma fecha, debido a su vinculación con el tema. El consejo destacó la pertenencia del Dr. Vidal al organismo y el área de estudio a la que se abocaba en su rol de investigador (refutando que se tratase de un investigador en oncología), pero señalaba no poseer información sobre el uso de productos derivados de complejos enzimáticos para el tratamiento del cáncer ("Controversias por la eficacia de una droga anticancerígena", 1986).

Ante el escepticismo y el asombro transmitidos por diferentes expertos y autoridades de asociaciones médicas oncológicas, los médicos Coni Molina, Costa y Hernández Plata adoptaron una posición en común: "No podemos echar cinco años de estudios a la basura. Aunque para nosotros esta difusión ha sido un parto prematuro, porque íbamos a publicar el descubrimiento - que se lo debemos pura y exclusivamente al doctor Vidal- una vez que se conociera en nuestro país" (“Controversias por la eficacia de una droga anticancerígena”, 1986). Los tres médicos expresaban la intención de "preservar el patrimonio nacional del descubrimiento" "Controversias por la eficacia de una droga anticancerígena", 1986). Esta intención se contraponía, estrictamente, a las "enormes presiones de laboratorios" que los médicos remarcaban haber sufrido (Barrios Medina \& Dellacha, 2007, p. 35). Pocos días después, la primera de una serie de manifestaciones en defensa de la crotoxina se sucedía en Plaza de Mayo frente a la casa de Gobierno (De Ípola, 2002, p. 393). En este contexto de ebullición, diputados, senadores, agrupaciones sociales y hasta la principal central obrera del país se pronunciaron en torno al caso. Varios frentes se habían abierto y una fuerte conmoción se había desatado en la esfera pública. El anuncio de un tratamiento antitumoral de gran eficacia, el desconocimiento general de la comunidad científica y de las autoridades gubernamentales y la denuncia de presiones en torno al patentamiento del descubrimiento conformaron en un primer momento los aspectos destacados del conflicto.

A medida que pasaban los días desde aquel miércoles 9 de julio de 1986, el conflicto iba incorporando a diversos actores. El msyAs, luego de la primera reunión con los médicos Coni Molina, Costa y Hernández Plata, tomó la decisión de conformar una comisión de oncólogos que estudiara la monografía que había sido presentada por los tres médicos en la reunión, quienes, además, habían solicitado la continuación de los ensayos clínicos.

La comisión del msyAs elevó un primer informe rápidamente, en el que rechazaba la solicitud de continuación de los ensayos clínicos que los tres médicos habían realizado argumentando que la información provista resultaba insuficiente para justificar su continuación (Braun, 1989, p. 72). El ministerio resolvió, por su parte, "autorizar el uso del compuesto a los pacientes que habían iniciado aplicaciones con anterioridad y bajo exclusiva responsabilidad de ellos mismos" (Braun, 1989, p. 72). El ministro Storani señaló que la decisión de autorizar la aplicación del complejo a 83 pacientes era de carácter político y social, e invocaba razones exclusivamente "humanitarias" (“Autorizan el suministro de la droga para 80 pacientes", 1986).

Para asegurar la producción del complejo, el ministro Storani recurrió al presidente del Conicet, Carlos Abeledo, quien constituyó una comisión ad hoc que estaría a cargo de examinar los antecedentes científicos basándose en la 


\section{DISERTACIONES}

ESTUDIOS

Comunicación y controversias sociocientíficas en salud y medio ambiente

ISSN: 1856-9536

Doi: https://doi.org/10.12804/revistas.urosario.edu.co/disertaciones/a.9774

Volumen 14, Número 2 / Julio-diciembre 2021

Versión PDF para imprimir desde

http://revistas.urosario.edu.co/index.php/disertaciones

monografía entregada por los tres médicos y en entrevistas a sus autores (Yriart \& Braginski, 1998, p. 117). Además, Abeledo encomendó a tres institutos de investigación en ciencias biomédicas la realización de ensayos in vitro y con animales, para verificar la postulada acción antitumoral y la toxicidad de la fracción de veneno de serpiente que había sido empleada en los ensayos clínicos realizados por los tres médicos y Vidal (Yriart \& Braginski, 1998, p. 118). Asimismo, el ministro Storani solicitó la asistencia de Juan H. Tramezzani para organizar la producción del complejo. Tramezzani reunió en el IDNEU a los representantes de la comisión del Conicet y a los colaboradores del Dr. Vidal. Durante la reunión consignada con la comisión, Canziani y Seki, dos de las colaboradoras de Vidal, informaron que el complejo contenía veneno de serpiente de cascabel, pero también veneno de cobra (Naja siamensis) (Barrios Medina \& Dellacha, 2007, pp. 59 y 60). En su siguiente informe, la comisión del Conicet dio cuenta de esto al señalar el problema que presentaba para la producción de la sustancia el hecho de que el complejo contenga veneno de un tipo de serpiente que no pertenecía a la fauna del país (“Tendría partes tóxicas la crotoxina”, 1986). Ante este descubrimiento y en medio de la enorme presión social, el msyAs ratificó su decisión de autorizar la crotoxina a los 83 pacientes a través de la Resolución 522. Para su cumplimiento, el ministerio constituyó una nueva comisión que, además, evaluaría y examinaría, junto a los tres médicos Costa, Coni Molina y Hernández Plata, los aspectos clínicos del tratamiento en los pacientes que lo continuaban (Yriart \& Braginski, 1998, p. 117).

A partir de la resolución ministerial, se generó un amplio número de solicitudes judiciales de acceso al tratamiento con el complejo crotoxina y su suministro por parte del Estado argentino, bajo la figura del recurso de amparo. Las solicitudes justificaban el pedido considerando el estado terminal de los enfermos ("Un recurso de amparo para que se autorice la aplicación de crotoxina", 1986). Por otro lado, en la defensa del pedido de acceso al tratamiento se encontraba la invocación al "derecho a la vida" asociado a la posibilidad de recibir un tratamiento sobre el que había sido planteado un efecto positivo ("Crotoxina”, 1986). Ambos argumentos configuraban una nueva dimensión sobre el conflicto, en rigor: un dilema relativo al campo de la ética y la política.

A fines de julio de 1986, el Dr. Juan Carlos Vidal arribaba al país luego de ser citado por el msyas y prestaba declaración con relación a una causa de recurso de amparo de una enferma oncológica. Durante un extenso interrogatorio, Vidal llegó a brindar un veredicto determinante sobre los beneficios y efectos del complejo crotoxina: "yo en ningún momento dije que esto cure", a la vez que rehusaba su implicancia en el conflicto y en la repercusión pública que este había generado (Barrios Medina \& Dellacha, 2007, p. 69). El 29 de julio, el Conicet publicó un comunicado a partir de las declaraciones de Juan Carlos Vidal. En el comunicado, el consejo destacaba que el Dr. Vidal no se encontraba en condiciones de aportar "datos, a nivel de investigación básica o clínica, que signifiquen evidencias acerca de los supuestos efectos beneficiosos de la droga en cancerología" ("Todavía existen serias dudas sobre el valor terapéutico de la crotoxina", 1986). En un principio alineado con el discurso del consejo, Vidal adoptó luego una postura crítica contra algunas autoridades del Conicet y especialmente contra su director, Carlos Abeledo. El consejo había dado cuenta de una serie de falsedades e inconsistencias en la monografía elaborada por Vidal y los tres médicos (Braun, 1989, p. 71). Denunciando un destrato por parte del Conicet y una "falta de idoneidad" por parte de los miembros de la comisión de ciencias médicas del consejo para "juzgar el tema de los venenos ofídicos", Vidal renunció a su cargo en el organismo y volvió a Estados Unidos ("Duras críticas al Conicet y a la comisión de oncólogos", 1986). A mediados de agosto, el Conicet inició un sumario administrativo con el objetivo de investigar el asunto y "deslindar responsabilidades" ("Duros términos del Conicet sobre la crotoxina", 1986). 


\section{DISERTACIONES}

ESTUDIOS

Comunicación y controversias sociocientificas en salud y medio ambiente

ISSN: 1856-9536

Doi: https://doi.org/10.12804/revistas.urosario.edu.co/disertaciones/a.9774

Volumen 14, Número 2 / Julio-diciembre 2021

Versión PDF para imprimir desde

http://revistas.urosario.edu.co/index.php/disertaciones

Entre tanto, enfermos oncológicos, pacientes y familiares de pacientes crearon la Comisión Crotoxina Esperanza de Vida, que pugnaba por el suministro de crotoxina para enfermos oncológicos (Barrios Medina \& Dellacha, 2007, p. 88). Los vastos testimonios, expuestos en medios de comunicación, de integrantes de las multitudes que manifestaban y de la Comisión Crotoxina Esperanza de Vida, en particular, parecían tornarse en la evidencia necesaria para demostrar la utilidad y la eficacia de la crotoxina. Inapelablemente, la crotoxina se había erigido como una luz de esperanza, habida cuenta de sus efectos en la calidad de vida, según estos testimonios (Barrios Medina \& Dellacha, 2007, p. 89). La contracara de esta percepción era el dramatismo con el que se vivían las decisiones gubernamentales y la prolongación en el tiempo de la investigación sobre el caso. A esto se sumaba una noción que circulaba acerca de que las objeciones a la eficacia de la crotoxina y a la idoneidad de los tres médicos investigadores "tenían origen en firmas multinacionales que, o bien pretendían proteger sus inversiones en las terapias convencionales, o bien querían ellas mismas apoderarse con exclusividad del medicamento" (De Ípola, 2002, p. 391).

Las investigaciones de las comisiones del Conicet y del ministerio continuaron durante los siguientes meses, pero el conflicto no se paralizó. Representantes gubernamentales y de la Comisión Crotoxina Esperanza de Vida fueron citados por una subcomisión del Congreso, en la que pacientes, enfermos y sus familiares denunciaron la opinión "sesgada" de los investigadores del Conicet y del msyAs, añadiendo que costaba mucho "volcar los paradigmas de la ciencia" (Barrios Medina \& Dellacha, 2007, p. 89). En medio de aquella atmósfera de desconfianza proyectada sobre el trabajo y las consideraciones de los especialistas, el 13 de octubre de 1986 la comisión de oncólogos del ministerio suministró su informe final: "luego de un trabajo intenso y profundo, podemos afirmar sin ninguna duda que, lamentablemente, el denominado Complejo Crotoxina A y B carece de toda acción anticancerosa comprobable clínicamente" (Brailovsky, 1986). Al día siguiente, el ministro Storani prohibía la producción, el suministro y la prescripción de crotoxina a través de la Resolución 47 ("Fue prohibido el uso de crotoxina", 1986). En consonancia con el informe de la comisión de oncólogos del msyAs, el Conicet inició una querella contra Vidal, Tramezzani, Costa, Coni Molina, Hernández Plata, Canziani y Cristina Seki por delitos contra la salud pública, entre otros (Barrios Medina \& Dellacha, 1986, p. 77).

Durante los meses posteriores, el conflicto alrededor de la crotoxina fue atemperándose y las expresiones públicas disminuyeron, a pesar de que las expectativas sociales permanecerían latentes (Pellegrini, 2019, p. 158). De todas maneras, la controversia no acabó allí. A fines de 1989, la crotoxina volvió a la esfera pública de la mano de un nuevo secretario de salud, el Dr. Raúl Matera, y en medio de prominentes cambios políticos y económicos que se desarrollaron a lo largo de la década de 1990. Las investigaciones oncológicas con crotoxina retornaron en dos etapas, esta vez, con el impulso y el respaldo gubernamentales durante buena parte de aquella década. Incluso, el Dr. Vidal volvió a Argentina y fue reincorporado al Conicet, luego de ser sobreseído en 1993 de los cargos iniciados por la misma institución (Barrios Medina \& Dellacha, 2007, pp. 111 y 112). Pero los resultados del atribuido efecto antitumoral no terminaban de aparecer. Un último intento fue llevado a cabo con resultados farmacológicos "promisorios" de un ensayo clínico que fueron informados en 1996 a la Administración Nacional de Medicamentos, Alimentos y Tecnología Médica, organismo que había autorizado el

1 La comisión de expertos pronunció su dictamen con el acuerdo de 11 de sus 12 integrantes (Barrios Medina \& Dellacha, 2007, p. 74). 


\section{DISERTACIONES}

ESTUDIOS

Comunicación y controversias sociocientificas en salud y medio ambiente

ISSN: 1856-9536

Doi: https://doi.org/10.12804/revistas.urosario.edu.co/disertaciones/a.9774

Volumen 14, Número 2 / Julio-diciembre 2021

Versión PDF para imprimir desde

http://revistas.urosario.edu.co/index.php/disertaciones

proyecto (Barrios Medina \& Dellacha, 2007, pp. 135-137). Los resultados incentivaron al Dr. Jorge Cura, director del estudio, a solicitar financiamiento; pero debido al conflicto generado con la Fundación de Mecanismos Citostáticos Antitumorales con Enzimas (fundada por integrantes de la Comisión Crotoxina Esperanza de Vida y el Dr. Vidal, entre otros), con la que Cura había establecido un convenio para el financiamiento de un posterior estudio, y la reticencia de esta a acudir a las autoridades gubernamentales por las vías institucionales para la continuación del proyecto, se suspendieron definitivamente los estudios con crotoxina en Argentina (Barrios Medina \& Dellacha, 2007, pp. 136 y 137).

\section{Los medios de comunicación en el caso de la crotoxina}

Anteriormente nos abocamos a la tarea de reconstruir la historia de la controversia sobre la crotoxina como posible tratamiento antitumoral en Argentina, durante las décadas de 1980 y 1990. La controversia atravesó diferentes etapas e involucró a varios y diversos actores en cada una de ellas; pero, sin duda, el punto que marcó el inicio del conflicto en la arena pública, la situación a partir de la cual se generó una repercusión de alcance masivo en el país, fue la aparición de los tres médicos en medios masivos de comunicación. La incursión del tema en la arena pública tuvo lugar, en primera instancia, a través de una conferencia de prensa y, en segundo lugar, en el programa de televisión La Noticia Rebelde. A través de ambas exposiciones no se procuró únicamente, ni principalmente, hacer público el descubrimiento y la investigación referidas por los tres médicos, sino presentar una protesta ante la negativa del IDNEU a continuar suministrando -como hasta ese momento- el complejo crotoxina (De Ípola, 2002, p. 374).

La conferencia de prensa fue el primer contacto documentado de los médicos con periodistas de medios de comunicación. Ocurrió el martes 8 de julio de 1986 y fue impulsada por la experiodista y artista plástica Martha Gavensky, quien había trabajado como corresponsal de la agencia France Presse y, por ello, poseía contactos con una importante cantidad de medios y periodistas (Barrios Medina \& Dellacha, 2007, p. 32). Gavensky, amiga y paciente del médico Carlos Coni Molina, afirmó en su libro Crotoxina. Informe y testimonios que aquel 8 de julio ella tomó la "responsabilidad de llamar a una conferencia de prensa" (1986, p. 93). La motivación de aquella empresa se basó, en palabras de Gavensky, en que era la única alternativa pues “había ochenta vidas en juego", en referencia a los pacientes que eran tratados por los tres médicos y Vidal con el complejo crotoxina (Barrios Medina \& Dellacha, 2007, p. 83). Hernández Plata, uno de los tres médicos, ahondó, en ese sentido, en la motivación que los había llevado a tomar la decisión de hacer público, a través de medios de comunicación, su investigación y su protesta tras la imposibilidad de continuar recibiendo el complejo crotoxina por parte del IDNEU:

Los pacientes se encontraban profundamente inquietos respecto del futuro de nuestro ensayo [...] Pero además de esta inquietud -que es muy delicada tratándose de un enfermo de cáncer, que así vuelve a ver amenazada su vida cuando había logrado recuperar la esperanza - los pacientes se ven "invadidos" [...] En otras palabras: manoseados. (Gavensky, 1986, p. 60)

Hernández Plata concluye, en sintonía con Gavensky, que "no hubo alternativa [...] Esta acción finalmente nos llevó a la resolución número 522, tomada de inmediato por el ministro Storani” (Gavensky, 1986, p. 64). Posteriormente, Gavensky afirmó su convicción de que "si el tema no fuera auténtico -como muchos han preferido suponer- la crotoxina estaría ya hoy traspapelada, olvidada en el oscuro mar de los argumentos apócrifos [...] 


\section{DISERTACIONES}

ESTUDIOS

Comunicación y controversias sociocientificas en salud y medio ambiente

ISSN: 1856-9536

Doi: https://doi.org/10.12804/revistas.urosario.edu.co/disertaciones/a.9774

Volumen 14, Número 2 / Julio-diciembre 2021

Versión PDF para imprimir desde

http://revistas.urosario.edu.co/index.php/disertaciones

En el tema crotoxina son los pacientes de cáncer quienes deberían tener la última palabra” (1986, pp. 93 y 94). En síntesis, la entrada a los medios de comunicación obedecía a la búsqueda por obtener visibilidad para hacer públicas sus demandas. El ingreso a los medios de comunicación es una de las acciones más frecuentemente adoptadas por actores sociales como forma de estrategia para obtener visibilidad en sus demandas y reclamos. Según Gamson y Wolfsfeld (1993, p. 116), volver un conflicto más público ofrece una oportunidad para aumentar el poder relativo de los actores frente a sus antagonistas, y los medios masivos de comunicación son un vehículo para ello. Así, los medios pueden transformarse en un aliado para generar simpatía pública hacia su posición. Bennet y Segerberg (2015, p. 371) destacan que el éxito de un movimiento social suele depender de recibir mensajes positivos a través del encuadre (framing) que sus reclamos y demandas tengan en los medios de comunicación.

En la exposición, los médicos anunciaron el descubrimiento de un tratamiento antineoplásico exitoso, narraron la historia de la investigación que culminó en este descubrimiento y explicaron las motivaciones que los llevaron a realizar esta presentación fuera del ámbito de la comunidad científica:

No queríamos hacer propaganda porque la producción de la droga en la actualidad no alcanzaría a abastecer toda la demanda real. Queríamos regular la información porque debíamos seguir las pautas informativas que la comunidad científica nos impone, pero ante el peligro de vida que corren los pacientes que están siendo investigados decidimos comunicar el descubrimiento. ("Descubren en el país una droga contra el cáncer", 1986)

De acuerdo con Barrios Medina y Dellacha (2007, p. 28), solo el periódico Tiempo Argentino asistió a la conferencia de prensa el 8 de julio de 1986. Al día siguiente, el matutino publicó la nota "Descubren en el país una droga contra el cáncer" (1986), en cuya bajada se explica que "se trata de un complejo enzimático, único en su tipo, altamente eficaz para combatir todos los tumores". La nota del periódico Tiempo Argentino releva la conferencia de prensa y cita las palabras de los médicos Coni Molina, Costa y Hernández Plata, quienes se explayan sobre el desarrollo de la investigación, los ensayos clínicos y los aspectos técnicos de la aplicación del complejo crotoxina (referido como "droga"), dan por sentada la eficacia del tratamiento (se cita al médico Guillermo Hernández Plata con la siguiente declaración, de manera textual: "no existe ningún tumor en especial que se resista a la droga") y, por último, explicitan el motivo del anuncio: "comunicamos hoy la existencia de este descubrimiento científico porque la institución que proveía a nuestros pacientes del producto se ha negado a seguir suministrándolos [...] tenemos 80 pacientes en tratamiento y el suministro del medicamento es indispensable" ("Descubren en el país una droga contra el cáncer”, 1986). Además, en la nota se añade el testimonio de un paciente de los médicos, a quien presentan por su rol profesional ("médico psiquiatra"), Mauricio Olemberg; a su vez, se destaca la implicancia sobre la posible pérdida económica que tendría para el país el abandono del descubrimiento anunciado: "La pérdida económica, de trascender la fórmula en otros países, equivaldría a entregar una provincia argentina" (“Descubren en el país una droga contra el cáncer", 1986). La información es presentada en el artículo periodístico de manera apodíctica, en el cual su única fuente es el anuncio de los médicos en la conferencia de prensa.

La segunda exposición mediática de los tres médicos tuvo lugar en el programa humorístico La Noticia Rebelde, el 9 de julio de 1986. La Noticia Rebelde era un programa de humor político, transmitido por ATC (Argentina Televisora Color) y conducido, en aquel entonces, por Jorge Guinzburg, Carlos Abrevaya, Raúl Becerra y Adolfo Castelo. En julio de 1986 gozaba de una audiencia de alrededor de 500000 hogares en Argentina (Barrios Medina \& Dellacha, 2007, p. 34). En la mañana del 9 de julio, el periodista y humorista Raúl Becerra, uno de los conductores 


\section{DISERTACIONES}

ESTUDIOS

Comunicación y controversias sociocientificas en salud y medio ambiente

ISSN: 1856-9536

Doi: https://doi.org/10.12804/revistas.urosario.edu.co/disertaciones/a.9774

Volumen 14, Número 2 / Julio-diciembre 2021

Versión PDF para imprimir desde

http://revistas.urosario.edu.co/index.php/disertaciones

de La Noticia Rebelde, solicitó a una de las productoras del programa, Gabriela Bruzos, que contactara "sí o sí, a los médicos de la crotoxina" (G. Bruzos, comunicación personal, 21 de septiembre de 2020). Becerra tenía la intención de conseguir una entrevista con los médicos para que presentaran sus hallazgos en el programa (G. Bruzos, comunicación personal, 21 de septiembre de 2020).

En su libro, Making the News, Golding y Elliot se preguntan acerca de "¿Qué imagen del mundo dan los informativos radiotelevisivos? ¿Cómo se relaciona esta imagen con las exigencias cotidianas de la producción de noticias en las organizaciones radiotelevisivas?" (1979, p. 1). Wolf (1987, pp. 214 y 215) sostiene que estas preguntas definen el ámbito y describen los problemas de los que se ocupa la perspectiva del newsmaking, uno de los estudios abocados a los procesos productivos en las comunicaciones de masas. Dentro de esta perspectiva, con puntos en contacto con la teoría del framing y la teoría del establecimiento de la agenda (agenda-setting), se destaca la noción de noticiabilidad como un conjunto de criterios de importancia a partir de los cuales se define la "aptitud" de un acontecimiento para ser transformado en noticia (Wolf, 1987, p. 222). ¿Qué criterios pudieron haber sido tenidos en cuenta en el momento de aventurarse a realizar una entrevista a tres médicos que afirmaban estar tratando "exitosamente" a sus pacientes oncológicos con un complejo enzimático? Sobre el motivo del interés de Becerra en realizar la entrevista, Bruzos consideró: "Becerra era publicista, además de periodista. [...] Con lo cual, creo que ese día vio el rating del programa. O sea, dijo 'la cura para el cáncer es el rating del programa"” (G. Bruzos, comunicación personal, 21 de septiembre de 2020). Wolf (1987, p. 224) distingue, como un componente de los criterios de noticiabilidad, los "valores/noticia" (news values).

Este componente aparece esquematizado en una serie de criterios específicos que operan, de manera complementaria en la práctica, en torno a la noción de noticiabilidad. Dentro de estos news values encontramos los criterios sustantivos que se articulan en relación con dos factores: "la importancia y el interés de la noticia" (Wolf, 1987, pp. 222-238). Aquí se encuentran, entre otros: el grado y nivel jerárquico de los actores implicados en el acontecimiento, el posible impacto sobre el interés nacional, la cantidad de personas implicadas en el acontecimiento (de hecho o potencialmente), la importancia y significatividad del acontecimiento respecto a la evolución futura de una determinada situación, la novedad entendida como la cercanía temporal de un acontecimiento y el momento en el que es informado.

La información disponible sobre la crotoxina, hasta ese momento, parecía cumplir con aquellos criterios: involucraba a médicos matriculados como referentes de autoridad en la materia, el "descubrimiento" de un tratamiento contra una de las enfermedades más comunes y dañinas que existen, una cantidad importante de personas implicadas en el acontecimiento (los pacientes que no recibían el suministro del complejo y los enfermos que podrían beneficiarse por ese tratamiento), la noción de que la noticia no había tenido aún una repercusión masiva y el impacto en el interés nacional de un posible patentamiento extranjero. Al respecto de este último punto, Bruzos recordó que el caso tuvo una gran repercusión, entre otros factores, porque el abordaje en las noticias no se caracterizó solo por referirse a un tratamiento contra el cáncer, "que era lo que a la gente le importaba", sino por relevar las declaraciones de Coni Molina, Costa y Hernández Plata acerca de su intención de realizar el patentamiento del tratamiento "como producto argentino y, supuestamente -decían ellos- los estaban cercando porque lo quería patentar alguien en Eevu. La noticia era el patentamiento, la pelea por el patentamiento" (G. Bruzos, comunicación personal, 21 de septiembre de 2020). 


\section{DISERTACIONES}

ESTUDIOS

Comunicación y controversias sociocientificas en salud y medio ambiente

ISSN: 1856-9536

Doi: https://doi.org/10.12804/revistas.urosario.edu.co/disertaciones/a.9774

Volumen 14, Número 2 / Julio-diciembre 2021

Versión PDF para imprimir desde

http://revistas.urosario.edu.co/index.php/disertaciones

En la noche del 9 de julio - fecha bisagra para la historia de este caso- fue emitida la exposición de los tres médicos en el programa, en la que anunciaban (de manera similar a la ocasión de la conferencia de prensa) que "trataban exitosamente a pacientes de cáncer con una droga curativa de muy bajo costo descubierta por el científico argentino Juan Carlos Vidal". Jorge Guinzburg, uno de los conductores del programa, recordó sobre aquel día que "al terminar el programa, teníamos a toda la gente a la puerta de ATc" (Barrios Medina \& Dellacha, 2007, pp. 34 y 35). La Noticia Rebelde se emitía en el horario pre-prime-time (entre las 19 y las 21 horas). Bruzos, una de las productoras del programa, señaló haberse ido del estudio a las 2:00 de la mañana de aquel día, debido a la enorme cantidad de llamados al canal de personas que solicitaban poder contactarse con los "médicos de la crotoxina" (G. Bruzos, comunicación personal, 21 de septiembre de 2020).

Barrios Medina y Dellacha (2007, pp. 39 y 40) relatan que el mismo miércoles 9 de julio, luego de la finalización del programa, los médicos fueron entrevistados por Fernando Bravo y Jorge Fadelli en el marco del programa Revista Cinco, un programa emitido por la estación Radio Continental y que era conducido, precisamente, por el renombrado locutor Fernando Bravo. En la entrevista, los tres médicos reforzaron la condición abrupta del anunció: "Nunca quisimos dar esto a la luz pública hasta no tener garantizado y haber hecho todos los estudios correspondientes, de acuerdo a los protocolos internacionales para el uso de drogas y la investigación en cáncer" (Barrios Medina \& Dellacha, 2007, p. 40). Pero, en este caso, presentaron una información distinta en cuanto a la cantidad de pacientes que eran tratados con el complejo (razón que es esgrimida como motivante del anuncio: la cantidad de pacientes afectados): 700 en comparación con los 80 que habían sido referidos en la conferencia de prensa relevada por el periódico Tiempo Argentino. En la entrevista, los médicos afirmaron que el tratamiento era gratuito (aunque con la aclaración de que no se comercializaba, pues pertenecía a una investigación) y que la "fase experimental" de la investigación había sido concluida. El conductor, Fernando Bravo, hizo una breve mención respecto de la pertinencia de solicitar las palabras de las autoridades del Conicet frente a las declaraciones de los médicos y aclaró que al día siguiente se ocuparían de contactar a dichas autoridades (Barrios Medina \& Dellacha, 2007, p. 39).

Al detenernos en este punto es útil retomar la observación que hace Goodell (1987, p. 590) y que mencionamos en la introducción, sobre el patrón de dependencia en una cantidad exigua de fuentes científicas que los medios suelen generar en el tratamiento de una controversia científica. Hasta aquí, precisamente, hemos visto que la presentación y circulación de información sobre la crotoxina y la investigación desarrollada por los médicos Costa, Coni Molina y Hernández Plata, y el Dr. Vidal, solo tuvo como fuente, en un primer momento, a los tres médicos.

El 11 de julio de 1986, los periódicos La Prensa, La Nación y Clarín, entre otros medios, publicaron sus primeras notas sobre el tema. Los diarios La Nación y La Prensa presentaron una caracterización del asunto distinta a Clarín: en los primeros dos periódicos el conflicto fue presentado como un debate, a través de la exposición de citas que expresaban posiciones contrarias; mientras que, en el caso de Clarín, solo se hizo referencia a las declaraciones de los tres médicos sobre el complejo crotoxina, enfatizando el carácter experimental de la investigación referida por ellos. El artículo de La Nación hizo referencia al carácter "controversial" del tema, a partir de definir que lo que era discutido era la "eficacia de una droga anticancerígena" ("Controversias por la eficacia de una droga anticancerígena", 1986). La Prensa hizo referencia a un "supuesto hallazgo" y también definió al complejo como "droga contra el cáncer" (“Citarán a médicos por el supuesto hallazgo de una droga contra el cáncer”, 1986). En el caso de Clarín, en su artículo se afirma la existencia de una "droga contra el cáncer" que "resultaría eficaz en el tratamiento de 


\section{DISERTACIONES}

ESTUDIOS

Comunicación y controversias sociocientificas en salud y medio ambiente

ISSN: 1856-9536

Doi: https://doi.org/10.12804/revistas.urosario.edu.co/disertaciones/a.9774

Volumen 14, Número 2 / Julio-diciembre 2021

Versión PDF para imprimir desde

http://revistas.urosario.edu.co/index.php/disertaciones

los tumores", subrayando, como se mencionó, el carácter experimental del tratamiento ("Informaron sobre la droga contra el cáncer experimentada en el país”, 1986). Si bien las definiciones que se exhiben en los artículos sobre el asunto exponen la caracterización del conflicto realizada por los periódicos, también el tratamiento y la exposición de la información permite inferir dicha caracterización. En los tres periódicos son citadas como fuente de información las expresiones y afirmaciones de los médicos Costa, Coni Molina y Hernández Plata. En el caso de los periódicos La Nación y La Prensa, en sendos artículos fechados el 11 de julio de 1986, son citados también comunicados de autoridades institucionales (del IDNEu, en el caso de La Nación, y del msyas, en el caso de La Prensa). El tratamiento del tema se reitera en sendos artículos de los dos periódicos: ante los comunicados institucionales se ofrece como contraposición las afirmaciones de al menos uno de los tres médicos sobre el tema que evidencian la exposición del conflicto como un debate. En el caso de Clarín, solo es presentada como fuente de información la palabra de los tres médicos.

\section{Consideraciones finales}

A lo largo del presente artículo hemos analizado cómo los medios de comunicación desempeñan un papel decisivo en el surgimiento de un conflicto sociocientífico en la arena pública. El caso de la controversia en torno a la crotoxina es un ejemplo contundente de este aspecto y permite ilustrar cómo una controversia científica es una "controversia creada" (Harker, 2015) a partir de la irrupción de un tema de un modo determinado en los medios masivos de comunicación, los cuales instalan así una agenda particular (Martin, 2014); también permite ejemplificar cómo los medios suelen generar un patrón de dependencia en relativamente pocas fuentes científicas (Goodell, 1987).

El alcance masivo y la repercusión generada por la irrupción de los tres médicos defensores de la crotoxina en los medios de comunicación desató el conflicto en la esfera pública. A partir de esas apariciones en los medios, diversos actores sociales tomaron firmes posiciones públicas con respecto a la crotoxina. Organismos gubernamentales como el msyAs o el Conicet tomaron, a su vez, posición en el asunto, a través de comités de expertos. El análisis del caso de la crotoxina ilustra una situación en la que un tema vinculado a la ciencia se instala en la agenda pública a través de los medios masivos de comunicación, y lo hace de un modo específico: es presentado como una investigación con resultados promisorios en la lucha contra el cáncer que es injustamente clausurada. A pesar de instalarse en la arena pública de ese modo, en el ámbito científico la investigación en cuestión tenía un desarrollo incierto y sin haber atravesado aún ninguna instancia de legitimación colectiva por parte de la comunidad científica. En cuanto a los científicos que son convocados por los medios de comunicación para expresarse sobre el tema, se trató mayormente de los tres médicos y actores sociales que promovían el uso de la crotoxina; esto es: los medios de comunicación amplificaron la voz de quienes sostenían una de las posiciones del conflicto, la de que la crotoxina sería una promisoria cura contra el cáncer. Sin embargo, la evaluación que la mayoría de la comunidad científica realizó sobre el tema era muy distinta: en efecto, a través de los diversos comités de expertos convocados para expedirse sobre el conflicto, los dictámenes concluían que la crotoxina no constituía una cura contra el cáncer e, incluso, ponía en riesgo a los pacientes.

Hemos planteado que la caracterización del rol de los medios de comunicación como iniciadores de una controversia sociocientífica puede realizarse teniendo en cuenta: 1) cómo llega el tema a instalarse en la agenda pública y bajo qué perspectiva se presenta el tema; 2) cuál es el grado de desarrollo y discusión que tenía ese tema 


\section{DISERTACIONES}

ESTUDIOS

en la comunidad científica antes de llegar a la arena pública; 3) cuán representativas son las voces científicas que aparecen en los medios de comunicación en relación con el tema controversial, y 4) cuál es la evaluación que hace la mayoría de la comunidad científica sobre el tema y si difiere con el modo en que se presenta la evaluación del tema en la arena pública. Analizado frente a estos aspectos, el caso de la crotoxina revela así una serie de coincidencias con los casos que mencionamos al comienzo del trabajo.

En efecto, tanto en este conflicto como en los que tuvieron como protagonistas al Dr. Pusztai y al Dr. Carrasco, una investigación científica que aún no había arribado a resultados validados por pares científicos es difundida masivamente gracias a que dichos actores acuden a medios masivos de comunicación para alertar sobre lo que consideran una injusticia cometida contra la sociedad. A su vez, los involucrados justifican el haber acudido a los medios masivos antes que a los académicos, al considerar que debían advertir a la población sobre algo que la afectaba. Esa aparición en los medios de comunicación inicia una controversia sociocientífica, y la inicia además amplificando una posición que no necesariamente era representativa de la comunidad científica, sino que de hecho fue fuertemente cuestionada por los expertos científicos.

En rigor, el tema es presentado como un problema público bajo la óptica de los actores que acceden a los medios de comunicación; los medios serían el canal que da lugar a la voz de determinados actores. Pero ese es precisamente el rol clave de los medios de comunicación en una controversia sociocientífica de este tipo: no editorializando una posición explícita propia, sino dejando en manos de unos pocos actores - pero presentados como la autoridad en el tema - la definición del problema científico. De ese modo, los medios de comunicación inician un conflicto público sobre un tema científico que antes no existía, al presentar una voz marginal en el campo científico como el exponente de una controversia. La voz de esos actores era marginal dentro del campo científico hasta la llegada al medio masivo de comunicación.

Consideramos que continuar con el estudio del rol de los medios de comunicación como iniciadores de controversias sociocientíficas puede ser relevante para comprender el modo en que se despliegan las percepciones, las actitudes y los conflictos públicos sobre temas de ciencia y tecnología.

\section{Agradecimientos}

A la Agencia Nacional de Promoción de la Investigación, el Desarrollo Tecnológico y la Innovación (Ministerio de Ciencia, Tecnología e Innovación, Argentina), por el financiamiento PICT-2018-1997 para el estudio de controversias científicas.

\section{Referencias}

1. Autorizan el suministro de la droga para 80 pacientes. (1986, 15 de julio). La Prensa, 5.

2. Barrios Medina, A., \& Dellacha, J. (2007). El caso crotoxina: Una historia social de la ciencia biomédica argentina. Editorial Académica Española.

3. Bennet, W. L., \& Segerberg, A. (2015). Communication in movements. En D. Della Porta \& M. Diani (Eds.), The Oxford handbook of social movements (pp. 367-382). Oxford University Press.

4. Blumer, H. (1971). Social problems as collective behavior. Social Problems, (18), 298-306. 


\section{DISERTACIONES}

ESTUDIOS

Comunicación y controversias sociocientificas en salud y medio ambiente

ISSN: 1856-9536

Doi: https://doi.org/10.12804/revistas.urosario.edu.co/disertaciones/a.9774

Volumen 14, Número 2 / Julio-diciembre 2021

Versión PDF para imprimir desde

http://revistas.urosario.edu.co/index.php/disertaciones

5. Borelli, M. (2011). Voces y silencios: La prensa argentina durante la dictadura militar (1976-1983). Perspectivas de la Comunicación, 4(1), 24-41.

6. Braganca, B., Badrinath, P., \& Ambrose, E. (1965). A highly selective carcinolytic agent isolated from cobra venom. Nature, 207(4996), 534-535.

7. Brailovsky, J. (1986, 12 de octubre). Punto final a una fallida esperanza. La Nación, 17.

8. Braun, R. (1989). Crotoxina. Ciencia Hoy, 1(4), 70-73.

9. Calmette, A., Sáenz, A., \& Costil, L. (1933). Effets du venin de cobra sur les greffes cancereuses et sur le cancer spontane (adeno-carcinome) de la souris. Comptes Rendus Hebdomadaires des Séances de l'Académie des Sciences, 197(3), 205-209.

10. Citarán a médicos por el supuesto hallazgo de una droga contra el cáncer. (1986, 11 de julio). La Prensa, 3.

11. Controversias por la eficacia de una droga anticancerígena. (1986, 11 de julio). La Nación, 7.

12. Cotte, C., Essenfeld-Yahr, E., \& Lairet, A. (1972). Effects of crotalus and bothrops venom on normal and malignant cells cultivated in vitro. Toxicon, 10, 157-161.

13. Crotoxina. (1986, 5 de agosto). La Prensa, 4.

14. De Ípola, E. (2002). Estrategias de la creencia en situaciones críticas: El cáncer y la crotoxina en Buenos Aires a mediados de los años ochenta. En D. Armus (Ed.), Entre médicos y curanderos: Cultura, historia y enfermedad en la América Latina Moderna (pp. 371-416). Grupo Editorial Norma.

15. Descubren en el país una droga contra el cáncer. (1986, 9 de julio). Tiempo Argentino, (s. p.).

16. Duras críticas al Conicet y a la comisión de oncólogos. (1986, 2 de septiembre). La Nación, 12.

17. Duros términos del Conicet sobre la crotoxina. (1986, 15 de agosto). La Nación, 6.

18. El doctor Avelino Barrio se inyectó hace años el veneno de víbora. (1986, 26 de julio). La Prensa, 5.

19. Essex, H., \& Priestley, J. (1931). Effect of rattlesnake venom on flexner-jobling's carcinoma in the white rat (Mus norvegicus albinus). Proceedings of the Society for Experimental Biology and Medicine, 28(5), 550-551.

20. Fue prohibido el uso de crotoxina. (1986, 14 de octubre). La Nación, 11.

21. Gamson, W., \& Wolfsfeld, G. (1993). Movements and media as interacting systems. Annals of the American Academy of Political and Social Science, (528), 114-125.

22. Garrahan, P. (1995). Crotoxina: ¿Quién le pone el cascabel al gato? EXAcTamente, 2(3), 14-17.

23. Gavensky, M. (1986). Crotoxina: Informe y testimonios. Ruy Díaz.

24. Golding, P., \& Elliot, P. (1979). Making the news. Longman.

25. Goodell, R. (1987). Role of mass media in scientific controversy. En H. T. Engelhardt Jr. \& A. L. Caplan (Eds.), Scientific controversies: Case studies in the resolution and closure of disputes in science and technology (pp. 585-597). Cambridge University Press.

26. Gusfield, J. (2014). La cultura de los problemas públicos. Siglo xxı.

27. Harker, D. (2015). Creating scientific controversies. Cambridge University Press.

28. Hilgartner, S., \& Bosk, C. L. (1988). The rise and fall of social problems: A public arenas model. The American Journal of Sociology, 94(1), 53-78.

29. Hubo fraude y ocultamiento en la preparación de la droga. (1986, 28 de julio). La Prensa, 3.

30. Igal, D. (2019). La Noticia Rebelde: Una biografía. Grupo Editorial Sur.

31. Informaron sobre la droga contra el cáncer experimentada en el país. (1986, 11 de julio). Clarín, s. p. 
32. Lewenstein, B. V. (1995). Science and the media. En S. Jasanoff, G. E. Markle, J. C. Petersen y T. Pinch (Eds.), Handbook of science and technology studies (pp. 343-360). Sage.

33. Ley 20464 (1973, 18 de junio). Boletín Nacional de la República Argentina, Buenos Aires, Argentina.

34. Martin, B. (2014). Controversy manual. Irene Publishing.

35. Monaelesser, A., \& Taguet, C. (1933). Le traitement des tumeurs et des algies par le venin de cobra. Bulletin de l'Académie nationale de médecine de Paris, 109, 371-377.

36. Pellegrini, P. A. (2019). La verdad fragmentada: Conflictos y certezas en el conocimiento. Argonauta.

37. Rödder, S., Franzen, M., \& Weingart, P. (2012). The sciences' media connection - public communication and its repercussions. Springer.

38. Schäfer, M. S. (2011). Sources, characteristics and effects of mass media communication on science: A review of the literature, current trends and areas for future research. Sociology Compass, 5(6), 399-412.

39. Schulz, P., \& Katime, I. (2003). Los fraudes científicos. Revista Iberoamericana de Polímeros, 4(2), 1-90.

40. Tendría partes tóxicas la crotoxina. (1986, 25 de julio). La Nación, s. p.

41. Todavía existen serias dudas sobre el valor terapéutico de la crotoxina. (1986, 29 de julio). La Nación, 10.

42. Un recurso de amparo para que se autorice la aplicación de crotoxina. (1986, 22 de julio). La Nación.

43. Wolf, M. (1987). La investigación de la comunicación de masas: Críticas y perspectivas. Paidós.

44. Yriart, M., \& Braginski, R. (1998). La resurrección del caso crotoxina (1989-1996): Ciencia, política y medios de comunicación. Redes, 5(11), 113-139. 